Morbidity and mortality in children and adolescents listed for heart transplantation

Frey, S ; Prêtre, René ; Stiasny, B ; Noll, G ; Ruschitzka, F ; Balmer, C

Posted at the Zurich Open Repository and Archive, University of Zurich ZORA URL: https://doi.org/10.5167/uzh-53089

Journal Article

Published Version

Originally published at:

Frey, S; Prêtre, René; Stiasny, B; Noll, G; Ruschitzka, F; Balmer, C (2011). Morbidity and mortality in children and adolescents listed for heart transplantation. Cardiovascular Medicine, 14(6):176-181. 


\title{
Morbidity and mortality in children and adolescents listed for heart transplantation
}

\author{
Sarah Frey ${ }^{a}$, René Prêtre ${ }^{b}$, Brian Stiasny ${ }^{a}$, Georg Nollc, Frank Ruschitzkac ${ }^{c}$, Christian Balmer ${ }^{a}$ \\ a Departement of Cardiology, University Hospital Zurich, Switzerland \\ b Cardiovascular Surgery, University Children's Hospital, University Hospital Zurich, Switzerland \\ c Departement of Cardiology, University Hospital Zurich, Switzerland
}

\section{Summary}

Aim: This study aimed to summarise our recent clinical experience with children and adolescents selected for heart transplantation with a focus on morbidity and mortality during the waiting time.

Methods: This was a single centre, retrospective study of all consecutive patients listed for heart transplantation between 1994 and 2008 who were less than 20 years old. Clinical parameters, ECG, echocardiography and catheter data as well as duration and complications of mechanical circulatory support, were reviewed from the date of listing to the date of transplantation or death.

Results: The study population consisted of 20 patients with a median age at the time of listing of 13.8 years (range 1-19.6 years). Cardiomyopathy was present in 17 patients (dilated 11, restrictive 3, hypertrophic 1 and unclassified 2) and a congenital heart defect was present in 3. In 13/20 patients, a significant arrhythmia was noted: ventricular fibrillation 2 , non sustained ventricular tachycardia 6 , supraventricular tachycardia 8 and complete atrioventricular block 4 events. A total of five patients died whilst on the waiting list. The median time to transplantation or death was 61 days (range 1-318 days). The estimated survival rate (Kaplan Meier) was 88,71 and $47 \%$ at 1, 3 and 5 months respectively. There was no difference between the patients who died on the waiting list and the patients who survived to transplantation regarding age, sex, heart failure, arrhythmia and echocardiographic findings. Patients with dilated cardiomyopathy were overrepresented in the group who survived to transplantation (13/15 versus $1 / 5, p 0.014)$. Mechanical circulatory support was used in $13 / 20$ patients for a median duration of 59 days (range 1-361 days) resulting in 18 events of complications: thrombosis or embo-

Funding / potential competing interests: No financial support and no other potential conflict of interest relevant to this article were reported. lia 6 , bleeding 9 , and significant infection or sepsis 3 events. In one patient, a clinically relevant hemisydrome persisted.

Conclusion: The survival to transplantation rate was acceptable in our cohort and is similar to other studies. There were relatively few deaths in patients with dilated cardiomyopathy. Otherwise, an adverse outcome cannot be predicted from clinical or haemodynamic data at the time of listing for transplantation. Thrombo-embolic events continue to be a major issue in patients with mechanical circulatory support.

Key words: paediatric heart transplantation; congenital heart disease; ventricular assist device; heart failure

\section{Introduction}

Paediatric heart transplantation has been performed since 1982 [1]. In the past 28 years, the practice of paediatric cardiac surgery has evolved dramatically and heart transplantation is now available as an attractive treatment option for children and adolescents with end-stage heart failure. The actual estimated transplant half life (the time at which $50 \%$ of recipients remain alive) is now better than the average half life in adults and ranges from 11.3 to 15.8 years with the best survival reported in infants [1-3].

One of the main issues in paediatric heart transplantation is the lack of appropriate donor organs [4]. During the waiting time, the patients are at an increased risk for a further deterioration of the heart, and for the occurrence of arrhythmia or infection. Some patients may need mechanical circulatory support as a relatively safe "bridge" to transplantation. Children listed for heart transplantation face the highest waiting list mortality out of all solid-organ transplant patients [5].

The aim of this study was to critically assess our current approach to paediatric patients with end-stage heart failure who were identified as candidates for

Correspondence:

Christian Balmer, MD

University Children's Hospital

Steinwiesstrasse 75

$\mathrm{CH}-8032$ Zurich

christian.balmer@kispi.uzh.ch 
heart transplantation, and to compare the morbidity during the waiting time until the time of transplantation or death, within the last years at our centre.

\section{Methods}

This was a retrospective study in a single tertiary care centre. All consecutive patients of less than 20 years of age who were listed for heart transplantation for any reason, between the years 1994 to 2008 were included in the study. Data were collected retrospectively from patient charts and nursing notes. Echocardiography measurements were retrieved from our electronic database.

For data collection, we focused on the waiting time, which was the time between the listing for transplantation until the time of removal from the waiting list due to transplantation, death or recovery. The pretransplant clinical condition and assessment as well as the early post transplant mortality were also included in the study. A special emphasis was put on patients with mechanical circulatory support regarding mode, duration and rate of complication.

Patients with cardiomyopathy were divided into five groups: dilated, hypertrophic, restrictive, right ventricular arrhythmogenic and unclassified cardiomyopathies (such as non-compaction), in accordance with the report of the WHO/ISFC task force [6].

The indication for a heart transplant was individually judged using a multidisciplinary team approach. The following general rules were applied: candidates for heart transplantation were patients with end-stage heart failure with an estimated life expectancy of less than six months from any aetiology without any other feasible medical or surgical treatment option, in the absence of a systemic disease, infection, stroke or recent pulmonary infarction. During the study period, it was our policy not to perform heart transplantations within the first year of life. The psychosocial background of patients was routinely evaluated by a spe-

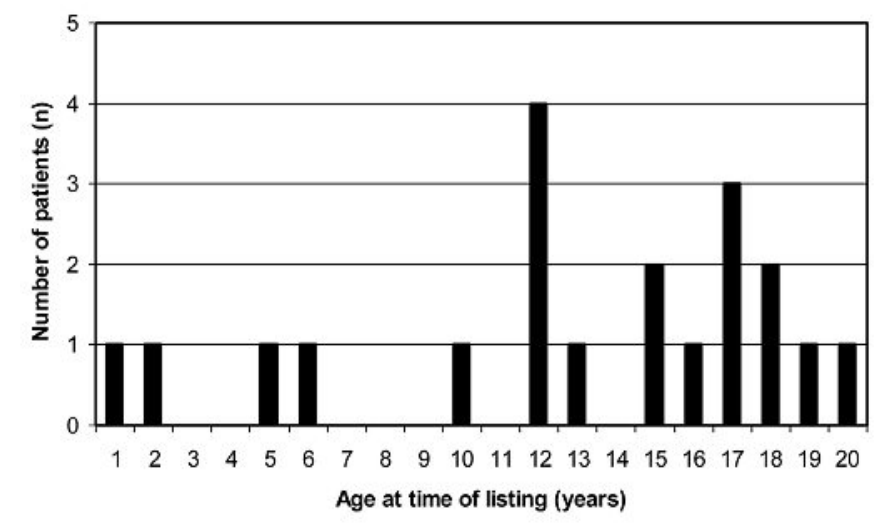

Figure 1

Age distribution at the time of listing for heart transplantation. cialised psychiatrist. A stable psychosocial background and evidence of strong motivation of the patient and the family were required. Donor and recipient were matched for ABO blood-type compatibility and body weight. A donor heart was obtained from a beatingheart brain dead individual through cooperation with the Swiss Transplant Organisation.

The routine diagnostic workup before heart transplantation included transthoracic echocardiography and right heart catheterisation, if possible. Due to the retrospective design of the study, the catheter datasets were incomplete and therefore they were not included in this paper. Pulmonary artery pressure was estimated echocardiographically by measurement of the pressure gradient between the right atrium and the right ventricle from the regurgitation of the tricuspid valve, and was graded relative to the simultaneously measured systolic blood pressure as mild (below half systemic pressure), moderate (equal or higher than half systemic pressure but below two thirds of the systemic pressure) or severe (equal or higher than two thirds of the systemic pressure).

Patients who survived the waiting time ("group survive") were compared with patients who died while waiting for heart transplantation ("group death"), to analyse for potential risk factors.

The study was approved by the local hospital ethical committee, and written informed consent was obtained for the data collection.

\section{Statistics}

Measurements are given as median and range. Patient groups were compared using the Mann-Whitney-test and Fishers-Exact-test. A significant difference was defined as $p$ value $<0.05$. Patient survival was calculated on the basis of the Kaplan Meier survival analysis. Echocardiographic measurements were compared using the $\mathrm{Z}$ score. For statistical analysis, we used SPSS version 14.0 for Windows.

\section{Results}

A total number of 20 patients younger than 20 years were listed at our institution during the study period of 15 years. There were 8 female and 12 male patients, and the median age at the time of listing for transplantation was 13.8 years (range 1-19.6). There was an asymmetric age distribution with only 4 patients aged 10 years or less (fig.1). The volume of cases per year varied from 0 to 3 patients. The median weight was $41 \mathrm{~kg}$ (range 10-94). Most but not all patients had severe symptoms of heart failure at the time of listing (NYHA 4: $\mathrm{n}=13$; NYHA 3: $\mathrm{n}=5$; NYHA 2: $\mathrm{n}=2$ [7]). The blood type of the listed patients was 0 in 7 patients, $\mathrm{A}$ in $9, \mathrm{~B}$ in 3 and $\mathrm{AB}$ in 1 patient.

Cardiomyopathy was the reason for heart failure in most of the patients (17/20; dilated 11 , restrictive 3 , 
hypertrophic 1 and unclassified 2). The aetiology of the dilated cardiomyopathy was as follows: myocarditis (5), muscular dystrophia (2), mitochondrial disease (1), anthracycline induced (1) and unknown (2). In 2 patients with dilated cardiomyopathy and myocarditis, Parvovirus B19 and Enterovirus were found in the endomyocardial biopsy as a possible aetiology in 1 patient each. Two patients had an unclassified cardiomyopathy, and their ventricular myocardium showed the typical aspect of a left ventricular non-compaction. Structural heart disease was seen in 3 patients, with 2 patients having a single ventricle. Both patients had left atrial isomerism and a severe stenosis of the pulmonary valve, palliated with systemic to pulmonary artery shunts. The third patient was postoperative atrial switch (Senning) for D-Transposition with intact ventricular septum and additional myocardial ischemia due to a thrombo-embolic obstruction of the right coronary artery.

Mechanical circulatory support was initiated in $13 / 20$ patients $(65 \%)$ because of a rapidly worsening clinical condition. A total of 10 patients were successfully bridged to transplantation, leading to a survival

\section{Table 1}

Description of technical data and events in patients with mechanical circulatory support (MCS); cumulative support time was 1018 days.

\begin{tabular}{lc} 
& $\begin{array}{c}\text { Number of patients } \\
\text { Total } \mathbf{n}=\mathbf{1 3}\end{array}$ \\
\hline Mode of MCS & 2 \\
\hline Biventricular & 10 \\
\hline Left ventricular only & 1 \\
\hline Right ventricular only & \\
\hline Complications during MCS & 3 \\
\hline Infection/Sepsis & 9 \\
\hline Bleeding & 6 \\
\hline Thrombosis/Embolia* & 8 \\
\hline Reoperations during MCS & 1 \\
\hline Revision for significant bleeding & 1 \\
\hline Cannula exchange & 1 \\
\hline Chamber exchange & \\
\hline Cannula replacement & \\
\hline 3 cerebral thrombo-embolic events in two patients, resulting \\
in a clinically relevant hemi syndrome in one patient
\end{tabular}

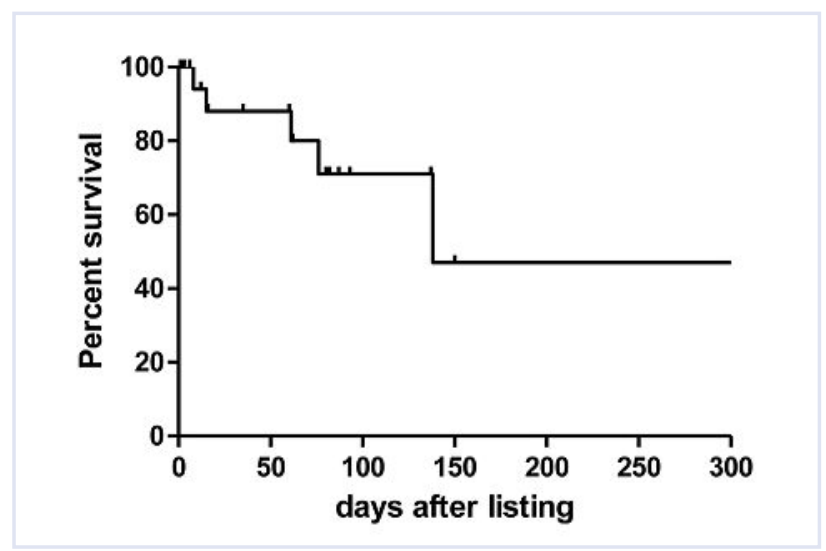

Figure 2

Freedom from death while waiting.

to hospital discharge rate of $62 \%$ (8/13) for all patients with mechanical circulatory support. The median duration of ventricular assist device in this cohort of 13 patients was 59 days, with a range of $1-361$ days. Various types of assist devices were used: pulsatile systems were used in 7 patients (Berlin Heart Excor 6, Abiomed 1) and non-pulsatile systems in 6 patients (DeBakey 5, Biomedicus 1). One patient had to be supported with extracorporal membrane oxygenation (ECMO) in addition to the implanted assist device for 20 days. The Berlin Heart Excor was the preferred device in the most recent patients. The total cumulative number of days on mechanical circulatory support was 1018. Events of all 13 patients who underwent mechanical circulatory support are listed in table 1 . The major complications were 3 thrombo-embolic neurologic events in 2 patients, leading to a clinically relevant hemi syndrome in one patient. There was no instance of technical failure of the blood pump components or of the driving systems.

\section{Clinical course during waiting time}

The median time of waiting was 61 days (range 1-318 days). A total of 14 patients finally underwent heart transplantation, 5 patients died whilst on the list (table 2) and 1 patient with dilated cardiomyopathy (DCM) recovered on the assist device and was discharged home on oral medication. The in-hospital survival rate of the whole study population of 20 patients

Table 2

Characteristics of patients who died during the waiting time (5/20).

\begin{tabular}{llllc} 
& Diagnosis & MCS & Cause of death & Time on waiting list (days) \\
\hline Patient 1 & RCM & No & Progressive atrial arrhythmia & 76 \\
\hline Patient 2 & HOCM & Yes & Sepsis & 138 \\
Patient 3 & Single ventricle & Yes & Ventricular fibrillation & 8 \\
\hline Patient 4 & RCM & Yes & Progressive atrial arrhythmia & 61 \\
\hline Patient 5 & DCM & No & Ventricular fibrillation & 15
\end{tabular}


Table 3

Patient characteristics; comparison of patients who died on the waiting list with patients who survived to transplantation (ns: $p>0.05$ ).

\begin{tabular}{|c|c|c|c|}
\hline & Died while waiting & Survived to HTPL & $p$ \\
\hline Number of patients (n) & 5 & 15 & \\
\hline Age (years); median (range) & $7.3(1.8-17.7)$ & $12.1(1-19.6)$ & ns \\
\hline Sex: female/male & $2 / 3$ & $6 / 9$ & ns \\
\hline Waiting time (days); median (range) & $61(8-138)$ & $61(1-318)$ & ns \\
\hline Dilated cardiomyopathy (n) & 1 & 13 & 0.014 \\
\hline Congenital heart defect & 1 & 1 & ns \\
\hline NYHA Class 4 (n) & 2 & 11 & ns \\
\hline $\begin{array}{l}\text { Ventricular function } \\
\text { EF (median) }\end{array}$ & 46 & 20 & ns \\
\hline Atrioventricular valve regurgitation moderate or severe $(n)$ & 2 & 10 & ns \\
\hline Aortic valve regurgitation moderate or severe $(n)$ & 0 & 1 & ns \\
\hline Inotropic support at time of listing & 2 & 10 & ns \\
\hline Mechanical ventilation at time of listing & 3 & 5 & ns \\
\hline Pulmonary hypertension moderate or severe $(n)$ & 3 & 10 & ns \\
\hline Mechanical circulatory support & 3 & 10 & ns \\
\hline ECMO & 1 & 1 & ns \\
\hline Arrhythmia & 5 & 8 & ns \\
\hline
\end{tabular}

was therefore $75 \%(15 / 20)$ (fig. 2). A total of three patients died early after heart transplantation from sepsis (2) and graft failure (1), resulting in a survival rate to hospital discharge of $60 \%$ (12/20). Demographic and clinical data was compared between the group of patients who died while waiting for an appropriate donor heart and the group who survived to transplantation (table 3).

Mechanical ventilation for progressive heart failure was necessary in $8 / 20$ patients at the time of listing, and one patient could be weaned from the respirator during the waiting time. Arrhythmia was noted in $13 / 20$ patients $(65 \%$; table 4$)$. Ventricular fibrillation was observed in $2 / 20$ patients. Every patient who died was affected by heart rhythm disturbances. None of the patients underwent preventative implantation of a cardioverter-defibrillator (ICD).

Echocardiography showed a decreased ventricular function in 19/20 patients. Median ejection fraction in patients with DCM was 20\% (range 5 to $40 \%$ ). Moderate or severe mitral valve regurgitation was seen in 4/11 and moderate or severe pulmonary hypertension in all 11 patients. The ejection fraction of the three patients with restricted cardiomyopathy (RCM) was a median of $46 \%(40-63 \%)$ at the time of indication and $40 \%(32-63 \%)$ at the time of transplantation. The patient with hypertrophic cardiomyopathy (HCM) had an ejection fraction of $46 \%$. Pulmonary artery hypertension was noted in 17/20 patients. It was assessed by echocardiography estimation as mild in $4 / 20$, moderate in $12 / 20$ and severe in $1 / 20$. A relevant mitral valve regurgitation in patients with two ventricles was present in five patients (moderate $3 / 13$ and severe 2/13). Of the two patients with a single ventricle, there was no relevant atrioventricular valve regurgitation.

All patients underwent repeated psychiatric counselling. One patient had suicidal ideation and another one had episodes of oppositional attitude. Continuous oral psychotropic medication was applied in 8/18 patients. The general qualitative psychological condition was good in 9 patients, mildly impaired in 5 and severely impaired in 4 patients. In two patients this data was not available.

\section{Table 4}

Arrhythmia observed on $24 \mathrm{~h}$ Holter monitoring at the evaluation for transplantation or during waiting time $(n=20$; multiple answers possible).

\section{No arrhythmia}

Supraventricular tachycardia

Atrial flutter 2

Atrial fibrillation 5

Non sustained AV reentrant tachycardia

Ventricular arrhythmia

Frequent isolated premature ventricular complexes 3

Non sustained ventricular tachycardia $\quad 6$

Ventricular fibrillation 2

Bradycardia

Sinus node dysfunction $\quad 2$

Complete heart block 4

Pacemaker for bradycardia 


\section{Discussion}

This paper gives a summary of our clinical experience with paediatric and adolescent candidates for heart transplantation within the last 15 years. Compared to other centres, the age distribution and underlying heart diseases of patients on the waiting list for heart tranplantation were similar to our study population $[8,9]$, whereas other centres, mainly from the United States, have a higher rate of transplantations in infants and younger children and also perform transplantations for relatively more patients with congenital heart defects [5, 10]. This discrepancy may be explained by our policy against infant heart transplantation and by our belief in reconstructive surgery for as long as possible in most end-stage heart failure patients, shifting the time of the operation towards teenagers and towards the group of patients with cardiomyopathies. Also, we did not offer heart transplantation to neonates with hypoplastic left heart syndromes or one of its variants, while other centres routinely performed heart transplantations in this patient group during this time period. Typically for the paediatric age group, ischemic heart disease is much rarer than in the adult cohorts and rare diseases such as restrictive cardiomyopathy or myocardial non compaction are overrepresented in this cohort. The most frequent type of cardiomyopathy in the current and other series was dilated cardiomyopathy [9, 11, 12].

Arrhythmia was a common finding among our study population (see table 5). In reviewing the individual cases, ventricular arrhythmia was seen in patients with advanced myocardial damage whereas patients with a predominant restrictive haemodynamic and dilated atria were seen to have supraventricular tachycardia.

The individual decision regarding the timing of heart transplantation is difficult in all patients but even more so in children and adolescents. In this age group, patients seem to cope well and for a relatively long time, if the progression of the disease is slow. Therefore it is difficult to predict further deterioration. Together with the constant lack of donor organs, we were relatively restrictive in listing a patient for heart transplantation. The fact that the survival to transplantation rate at our centre $(75 \%)$ was similar to that of other centres $(67-82 \%)[3,5,12,13]$ and that morbidity and mortality rates were comparable [3, 14, 15], indicates a similar state of the progress of the disease at the time of listing and hence similar listing criteria in other centres. This encourages us to continue with our current approach in children and adolescents with end stage heart failure.

The waiting time in other centres has been reported to range from a median duration of 7 days to 5 months [3, 12, 16, 17]. According to the current data, we calculated a median waiting time of 61 days in the current era. In 2007, a new law was introduced in Switzerland with the aim to improve the process of the allocation of donor organs and a new allocation system has been introduced which may shorten the waiting time substantially in the future [18]. In children, the coordination and allocation of donor organs also improved in 2009 due to the European Children Heart list, in which all members of the European Organ Exchange Organisation are able to list their patients under the age of twelve years.

Regarding waiting list mortality, dilatative cardiomyopathy was more common within the survivors of our cohort (table 4). This observation has been confirmed in other series with the exception of patients on ECMO, on mechanical ventilation or with severe arrhythmias [19].

In our series, a relative high number of patients required mechanical circulatory support (MCS) $(13 / 20=$ 65\%) when compared to another recently published series with the use of MCS in only $13 \%$ of the patients [20]. This may be due to a relatively generous indication of MCS mainly in the early years of our study. The technical progress of MCS within the last years has opened up this treatment option for the paediatric age group and even for infants [21]. However, it is still associated with a relevant morbidity and mortality. To minimise technical errors and because of the favourable experience in the paediatric age group [22], in the recent era we concentrated on one single pulsatile system. In MCS patients we were able to reach an acceptable survival to hospital discharge rate of $62 \%$. MCS has been used in other centres with similar success rates [23, 24]. Thrombo-embolic complications with neurologic sequelae were the most common relevant long term problems and continue to be a major issue. These complications are well known and occur in 6 to $32 \%$ of the patients in recently published series $[15,16$, $25]$.

\section{Conclusion}

Our current approach to children and adolescents with end-stage heart failure is in line with other centres. Arrhythmia was a common finding in our population. The overall morbidity and mortality during the waiting time was acceptable. A relatively high number of patients needed MCS. This did not result in an increased waiting list mortality however. MCS is feasible in this age group but should only be used in well selected patients because of the additional risk of complications such as thrombo-embolia.

\section{Limitations of the study}

The power of this analysis is limited due to the retrospective study design and the relatively small number of patients. The comparison of our findings with published data from other centres is hindered because of a 
possible selection bias regarding patients' age, underlying diagnosis and listing criteria.

Acknowledgement: We would like to thank Urs Bauersfeld, who sadly died so early, for his tremendous work and for his continuous support of the idea of the paediatric heart transplantation program in Zurich.

\section{References}

1 Kirk R et al. Registry of the International Society for Heart and Lung Transplantation: eleventh official pediatric heart transplantation report - 2008. J Heart Lung Transplant. 2008;27(9):970-7.

2 Fynn-Thompson F, Almond C. Pediatric ventricular assist devices. Pediatr Cardiol. 2007;28(2):149-55.

3 Tjang YS et al. Heart transplantation in children: clinical outcomes in a single center. Ann Thorac Surg. 2007;84(5):1640-4.

4 Dapper $\mathrm{F}$ et al. Clinical experience with heart transplantation in infants. Eur J Cardiothorac Surg. 1998;14(1):1-5; discussion 5-6.

5 Almond CS et al. Waiting list mortality among children listed for heart transplantation in the United States. Circulation. 2009;119(5):717-27.

6 Report of the WHO/ISFC task force on the definition and classification of cardiomyopathies. Br Heart J. 1980;44(6):672-3.

7 Towbin JA et al. Incidence, causes, and outcomes of dilated cardiomyopathy in children. Jama. 2006;296(15):1867-76.

$8 \mathrm{Lin} \mathrm{MH}$ et al. Outcome in children bridged and nonbridged to cardiac transplantation. Transplant Proc. 2010;42(3): 916-9.

$9 \mathrm{Chi} \mathrm{NH}$ et al. Outcome for pediatric cardiac transplantation with and without bridge methods. Asaio J. 2007;53(2):241-5.

10 Kirk $R$ et al. The Registry of the International Society for Heart and Lung Transplantation: thirteenth official pediatric heart transplantation report - 2010. J Heart Lung Transplant. 2010;29(10):1119-28.

11 Januszewska K et al. Ventricular assist device as a bridge to heart transplantation in children. Interact Cardiovasc Thorac Surg. 2009; 9(5):807-10.

12 McGiffin DC et al. Predicting outcome after listing for heart transplantation in children: comparison of Kaplan-Meier and parametric competing risk analysis. Pediatric Heart Transplant Study Group. J Heart Lung Transplant. 1997;16(7):713-22.
13 Shapiro PA, Levin HR, Oz MC. Left ventricular assist devices. Psychosocial burden and implications for heart transplant programs. Gen Hosp Psychiatry. 1996;18(6 Suppl):30S-35S

14 Gabrys CA. Pediatric cardiac transplants: a clinical update. J Pediatr Nurs. 2005;20(2):139-43.

15 Ruiz-Cano MJ et al. Successful heart transplantation in patients with inherited myopathies associated with end-stage cardiomyopathy. Transplant Proc. 2003;35(4):1513-5.

16 Goldman AP et al. The waiting game: bridging to paediatric heart transplantation. Lancet. 2003;362(9400):1967-70.

17 Blume ED et al. Outcomes of children bridged to heart transplantation with ventricular assist devices: a multi-institutional study. Circulation. 2006;113(19):2313-9.

18 Uehlinger NB et al. Organ transplantation in Switzerland: impact of the new transplant law on cold ischaemia time and organ transports. Swiss Med Wkly. 2010; 140(15-16):222-7.

19 Kirk $\mathrm{R}$ et al. Outcome of pediatric patients with dilated cardiomyopathy listed for transplant: a multi-institutional study. J Heart Lung Transplant. 2009;28(12):1322-8.

20 Jacobs JP et al. Lessons learned from 119 consecutive cardiac transplants for pediatric and congenital heart disease. Ann Thorac Surg. 2011;91(4):1248-55.

21 Karimova A et al. Mechanical bridging to orthotopic heart transplantation in children weighing less than 10kg: feasibility and limitations. Eur J Cardiothorac Surg. 2011;39:304-9.

22 Stiller B et al. Management of pediatric patients after implantation of the Berlin Heart EXCOR ventricular assist device. Asaio J. 2006;52(5):497-500.

23 Gajarski RJ et al. Use of extracorporeal life support as a bridge to pediatric cardiac transplantation. J Heart Lung Transplant. 2003;22(1):2834.

24 Gazit AZ, Gandhi SK, C Canter C. Mechanical circulatory support of the critically ill child awaiting heart transplantation. Curr Cardiol Rev. 2010;6(1):46-53.

25 Cassidy J et al. Changing patterns of bridging to heart transplantation in children. J Heart Lung Transplant. 2009;28(3):249-54. 